\title{
Hype and hope of hepatic arterial infusion for colorectal cancer
}

\author{
François Ghiringhelli ${ }^{1,2,3,4,5}$ \\ ${ }^{1}$ Centre de Recherche INSERM LNC-UMR1231, Dijon, France; ${ }^{2}$ University of Burgundy Franche-Comté, Dijon, France; ${ }^{3}$ Department of Medical \\ Oncology, Centre Georges-François Leclerc, Dijon, France; ${ }^{4}$ Cancer Biology Transfer Platform, Centre Georges-François Leclerc, Dijon, France; \\ ${ }^{5}$ Genetic and Immunology Medical Institute, Dijon, France \\ Correspondence to: François Ghiringhelli. Centre de Recherche INSERM LNC-UMR1231, Dijon, France. Email: fghiringhelli@cgfl.fr. \\ Comment on: Gholami S, Kemeny NE, Boucher TM, et al. Adjuvant hepatic artery infusion chemotherapy is associated with improved survival \\ regardless of KRAS mutation status in patients with resected colorectal liver metastases: a retrospective analysis of 674 patients. Ann Surg \\ 2020;272:352-6.
}

Submitted Oct 15, 2020. Accepted for publication Oct 28, 2020.

doi: $10.21037 / \mathrm{hbsn}-20-756$

View this article at: http://dx.doi.org/10.21037/hbsn-20-756

Contrary to most solid metastatic cancers, metastatic colorectal cancer could be cured by surgical removal of the metastasis in the context of lung or liver metastases. Large retrospective studies performed 20 years ago showed that surgery alone led to cure of the patient in about $35 \%$ of cases (1). To improve patient survival and to increase the cure rate, perioperative intravenous chemotherapy was proposed. In a large EORTC trial among highly selected patients, perioperative FOLFOX regimen improved relapse-free survival with an absolute difference in 3-year PFS of $8.1 \%$ (28.1\% in the surgery-only group $v s .36 .2 \%$ in the perioperative chemotherapy group) and there was a non-significant increase in overall survival (5-year overall survival was $51.2 \%$ in the perioperative chemotherapy group vs. $47.8 \%$ in the surgery-only group) (2). Despite this somewhat disappointing result, perioperative FOLFOX regimen became the standard of care for the management of resectable colorectal liver metastasis.

In the search to better stratify the risk of relapse, many clinical and pathologic factors, such as carcinoembryonic antigen, node positivity for the primary tumor, size and number of lesions, time interval between primary resection and the diagnosis of metastases, have been reported to predict the recurrence rate. Rat sarcoma viral oncogene homolog (RAS) mutations are detected in around $40 \%$ of colorectal cancers. RAS mutations are factors of resistance to anti EGFR therapies, and are associated with poor prognosis in the adjuvant and metastatic settings. Not surprisingly, the presence of RAS mutations has been associated with poor outcome, with reported 3-year overall survival rates of $81 \%$ vs. $52.2 \%$, and 3 -year recurrence-free survival rates of $33.5 \%$ vs. $13.5 \%$ for wild-type $v s$. mutant RAS tumors in the setting of liver metastasis surgery (3).

Because liver recurrence occurs in $50 \%$ of cases after liver metastasis surgery, it seemed logical to hypothesize that an increase in chemotherapy delivery to the liver might improve eradication of micro metastatic liver disease. Metastases in the range of 2 to $3 \mathrm{~mm}$ are not detectable, but could be responsible for liver relapse. Once a metastatic site exceeds $1 \mathrm{~mm}$ in size, hepatic metastases derive their blood supply from the hepatic artery, while normal hepatocytes are perfused mostly from the portal circulation. Therefore, adjuvant chemotherapy via the arterial circulation should logically increase drug delivery to undetectable liver metastasis. In the setting of colorectal cancer resistant to conventional chemotherapy, Hepatic Arterial infusion (HAI) using floxuridine yielded high response rates in multithreaded patients (4). Oxaliplatin can also be given by $\mathrm{HAI}$ and has shown considerable efficacy in association with intravenous perfusion of fluoropyrimidine or intraarterial infusion of raltitrexed $(5,6)$.

In a retrospective analysis generated from the MSKCC database of patients treated by surgery for liver metastases of colorectal cancer, Gholami et al. (7) report the follow up of 674 patients with known KRAS status [418 KRAS wild-type (WT), 256 KRAS-mutation (MUT)]. Fifty-four percent received HAI chemotherapy, in the adjuvant setting for the majority. Patients treated with adjuvant HAI and systemic therapy had a lower initial recurrence rate involving the liver than patients treated with adjuvant systemic therapy 
only $(28.4 \%$ vs. $35.8 \%, \mathrm{P}<0.04)$. Five-year relapse free survival (RFS) for patients with KRAS-WT was 33\% (38\% for patients treated with HAI plus systemic chemotherapy and $29 \%$ for patients treated with systemic chemotherapy only). For patients with KRAS-Mutant tumor, 5-year RFS was $23 \%$ (29\% for patients treated with HAI plus systemic chemotherapy $v s .18 \%$ for patients treated with systemic chemotherapy only). Five-year OS for patients with KRASWT was $69 \%$ ( $73 \%$ vs. $63 \%$ for patients treated with HAI plus systemic chemotherapy $v s$. systemic chemotherapy alone) and $50 \%$ in KRAS-Mutated patients (57\% for patients treated with HAI plus systemic chemotherapy $v s .43 \%$ for patients treated with systemic chemotherapy only). These data support the rationale that HAI given with systemic chemotherapy is efficient in enhancing RFS and OS, in both RAS wild-type and mutated tumors. This evidence also supports the use of HAI as a means to limit the poor prognosis of RAS mutated tumors.

Despite the impressive results reported from this retrospective single-centre study, caution is still warranted before the use of adjuvant HAI procedures. Only one old randomized clinical trial with small number of patients compared HAI with floxuridine vs. no adjuvant therapy. This study showed improved RFS, with no significant improvement in OS (8). The benefit is not very impressive in comparison with FOLFOX systemic adjuvant therapy, while the procedure is complex with a major risk of complication. Likewise, a recent Japanese phase III clinical trial failed to demonstrate the superiority of adjuvant HAI in comparison with intravenous chemotherapy, and reported substantial complication rates due to the procedure (9). Thus, based on current randomized clinical trials, adjuvant HAI should not be considered as a standard of care. Some ongoing clinical trials are currently addressing this question using different types of HAI procedure (NCT02494973, NCT02494973).

A major problem with the use of the HAI procedure after liver metastasis resection is extrahepatic relapse. Notably, lung recurrence occurs in around $50 \%$ of patients treated by liver metastasis resection (10). The presence of KRAS mutations was associated with an increased incidence of recurrence in the lung, bone and brain compared to wildtype patients (10), thus suggesting that the main issue in such tumors is not liver recurrence, but rather, systemic disease. With the development of triplet chemotherapy (11), which demonstrated a high rate of efficacy in metastatic disease in first line treatment, and the discovery that immunotherapy could be effective in the neoadjuvant setting for localized microsatellite stable colorectal cancer (12), there is reason to suspect that these therapies may take place and supplanted HAI in the setting of management of colorectal liver metastases.

\section{Acknowledgments}

We thank Fiona Ecarnot, PhD (EA3920, University of Franche-Comté, Besancon, France) for careful reading of the manuscript.

Funding: None.

\section{Footnote}

Provenance and Peer Review: This article was commissioned by the editorial office, Hepatobiliary Surgery and Nutrition. The article did not undergo external peer review.

Conflicts of Interest: The author has completed the ICMJE uniform disclosure form (available at https://hbsn. amegroups.com/article/view/10.21037/hbsn-20-756/coif). The author has no conflicts of interest to declare.

Ethical Statement: The author is accountable for all aspects of the work in ensuring that questions related to the accuracy or integrity of any part of the work are appropriately investigated and resolved.

Open Access Statement: This is an Open Access article distributed in accordance with the Creative Commons Attribution-NonCommercial-NoDerivs 4.0 International License (CC BY-NC-ND 4.0), which permits the noncommercial replication and distribution of the article with the strict proviso that no changes or edits are made and the original work is properly cited (including links to both the formal publication through the relevant DOI and the license). See: https://creativecommons.org/licenses/by-nc-nd/4.0/.

\section{References}

1. Fong Y, Fortner J, Sun RL, Brennan MF, Blumgart LH. Clinical score for predicting recurrence after hepatic resection for metastatic colorectal cancer: analysis of 1001 consecutive cases. Ann Surg 1999;230:309-18; discussion $318-21$.

2. Nordlinger B, Sorbye H, Glimelius B, et al. Perioperative FOLFOX4 chemotherapy and surgery versus surgery alone for resectable liver metastases from colorectal cancer 
(EORTC 40983): long-term results of a randomised, controlled, phase 3 trial. Lancet Oncol 2013;14:1208-15.

3. Vauthey JN, Zimmitti G, Kopetz SE, et al. RAS mutation status predicts survival and patterns of recurrence in patients undergoing hepatectomy for colorectal liver metastases. Ann Surg 2013;258:619-26; discussion 626-7.

4. Kemeny NE, Niedzwiecki D, Hollis DR, et al. Hepatic arterial infusion versus systemic therapy for hepatic metastases from colorectal cancer: a randomized trial of efficacy, quality of life, and molecular markers (CALGB 9481). J Clin Oncol 2006;24:1395-403.

5. Boige V, Malka D, Elias D, et al. Hepatic arterial infusion of oxaliplatin and intravenous LV5FU2 in unresectable liver metastases from colorectal cancer after systemic chemotherapy failure. Ann Surg Oncol 2008;15:219-26.

6. Ghiringhelli F, Vincent J, Bengrine L, et al. Hepatic arterial chemotherapy with raltitrexed and oxaliplatin versus standard chemotherapy in unresectable liver metastases from colorectal cancer after conventional chemotherapy failure (HEARTO): a randomized phase-II study. J Cancer Res Clin Oncol 2019;145:2357-63.

7. Gholami S, Kemeny NE, Boucher TM, et al. Adjuvant hepatic artery infusion chemotherapy is associated with improved survival regardless of KRAS mutation status in patients with resected colorectal liver metastases: a retrospective analysis of 674 patients. Ann Surg

Cite this article as: Ghiringhelli F. Hype and hope of hepatic arterial infusion for colorectal cancer. HepatoBiliary Surg Nutr 2021;10(2):235-237. doi: 10.21037/hbsn-20-756
2020;272:352-6.

8. Kemeny MM, Adak S, Gray B, et al. Combined-modality treatment for resectable metastatic colorectal carcinoma to the liver: surgical resection of hepatic metastases in combination with continuous infusion of chemotherapy-an intergroup study. J Clin Oncol 2002;20:1499-505.

9. Kusano M, Honda M, Okabayashi K, et al. Randomized controlled Phase III study comparing hepatic arterial infusion with systemic chemotherapy after curative resection for liver metastasis of colorectal carcinoma: JFMC 29-0003. J Cancer Res Ther 2017;13:84-90.

10. Kemeny NE, Chou JF, Capanu M, et al. KRAS mutation influences recurrence patterns in patients undergoing hepatic resection of colorectal metastases. Cancer 2014;120:3965-71.

11. Cremolini C, Antoniotti C, Rossini D, et al. Upfront FOLFOXIRI plus bevacizumab and reintroduction after progression versus mFOLFOX6 plus bevacizumab followed by FOLFIRI plus bevacizumab in the treatment of patients with metastatic colorectal cancer (TRIBE2): a multicentre, open-label, phase 3, randomised, controlled trial. Lancet Oncol 2020;21:497-507.

12. Chalabi M, Fanchi LF, Dijkstra KK, et al. Neoadjuvant immunotherapy leads to pathological responses in MMRproficient and MMR-deficient early-stage colon cancers. Nat Med 2020;26:566-76. 